\title{
Embryonic and extraembryonic stem cell lines derived from single mouse blastomeres
}

\author{
Young Chung ${ }^{1 \star}$, Irina Klimanskaya ${ }^{1 \star}$, Sandy Becker ${ }^{1 \star}$, Joel Marh ${ }^{1}$, Shi-Jiang Lu ${ }^{1}$, Julie Johnson ${ }^{2}$, \\ Lorraine Meisner $^{2,3} \&$ Robert Lanza ${ }^{1,4}$
}

The most basic objection to human embryonic stem (ES) cell research is rooted in the fact that $\mathrm{ES}$ cell derivation deprives embryos of any further potential to develop into a complete human being ${ }^{1,2}$. ES cell lines are conventionally isolated from the inner cell mass of blastocysts ${ }^{3-5}$ and, in a few instances, from cleavage stage embryos ${ }^{6-9}$. So far, there have been no reports in the literature of stem cell lines derived using an approach that does not require embryo destruction. Here we report an alternative method of establishing ES cell lines-using a technique of singlecell embryo biopsy similar to that used in pre-implantation genetic diagnosis of genetic defects ${ }^{10}$-that does not interfere with the developmental potential of embryos. Five putative ES and seven trophoblast stem (TS) cell lines were produced from single blastomeres, which maintained normal karyotype and markers of pluripotency or TS cells for up to more than $\mathbf{5 0}$ passages. The ES cells differentiated into derivatives of all three germ layers in vitro and in teratomas, and showed germ line transmission. Single-blastomere-biopsied embryos developed to term without a reduction in their developmental capacity. The ability to generate human ES cells without the destruction of ex utero embryos would reduce or eliminate the ethical concerns of many.

A series of six separate experiments was carried out to determine whether stem cell lines can be generated from single blastomeres (Supplementary Table 1). Eight-cell stage 129/Sv-ROSA26:lacZ mouse embryos were biopsied through a hole made in the zona pellucida with piezo-pulse drilling, and the biopsied (7-cell) embryos transferred to the oviducts of 1.5 days post coitum (d.p.c.) synchronized surrogate mothers. Each isolated blastomere was aggregated with a small clump of green fluorescent protein (GFP)-positive 129Sv/CD-1 mouse ES (mES) cells, and after incubation for 24-48 h, a growing 'bud' of GFP-negative cells was observed on the sides of the majority (60\%) of GFP-mES clusters (Fig. 1a, b). The cell aggregates were plated onto mitomycin C-treated mouse embryonic fibroblasts (MEFs) and cultured in mES cell growth medium ${ }^{11}$. Approximately half (36 out of 75 ) of them formed rapidly growing clumps of cells within 2-4 days, which were separated from GFPpositive $\mathrm{mES}$ cells by hand under a fluorescence microscope. The cells were expanded using mechanical and enzymatic methods, while further selecting by eye for the colonies morphologically resembling ES cells and excluding any GFP-positive cells (Fig. 1c-f). In four experiments, putative lines of $\mathrm{LacZ}^{+} \mathrm{ES}$ cells were produced (Fig. 2a, c) that exhibited normal karyotype (Fig. 3g) and maintained markers of pluripotency after up to $>50$ passages. Each line expresses octamer binding protein 4 (Oct-4), stage-specific embryonic antigen (SSEA)-1, nanog and alkaline phosphatase (Fig. 2e, g, i).
Polymerase chain reaction (PCR) analysis confirmed the presence of LacZ but not GFP gene sequences in these cells (Supplementary Fig. $1 \mathrm{a}, \mathrm{b})$. Together with the karyotype analyses, the absence of GFP also rules out the possibility of contamination and/or fusion of the blastomere-derived lines with the ES cells used for co-culture.

When the putative ES cell cultures were allowed to overgrow or form embryoid bodies, they readily differentiated into cells of all three germ layers, as evidenced by immunostaining with antibodies to muscle actin (mesoderm, Fig. 3a), $\alpha$-feto protein (primitive endoderm, Fig. 3c) and $\beta$ III tubulin (ectoderm, Fig. 3e). Beating heart muscle, extraembryonic endoderm and multiple neuronal cell types were also routinely observed in differentiating cultures. To demonstrate further the pluripotency of the derived putative ES cells, cells were either injected into CD-1 mouse blastocysts or aggregated with 8 -cell stage morulae as described ${ }^{11}$. Forty-eight injected/ aggregated embryos (between 8 and 15 per cell line) were transferred to recipient females. 5-bromo-4-chloro-3-indolyl- $\beta$-D-galactoside (X-gal) staining of the resulting 29 fetuses (followed from midgestation through to term) showed that the ES cell lines contributed to all organ systems, including heart, kidney, liver, lung, intestine, brain, blood, skin and genital ridge, among others. Twenty-four of the fetuses (83\%) were chimaeric (Fig. 3d, f), and eight out of nine (89\%) pups (Fig. 3h) were chimaeric; the latter had the LacZ gene in their gametes (confirmed by PCR analysis; Supplementary Fig. 3), and produced $\mathrm{LacZ}^{+}$offspring when crossed with CD-1 females, confirming the contribution of the blastomere-derived ES cells to the germ line. When the ES cells were injected into NOD-SCID (nonobese diabetic-severe combined immunodeficiency) mice, they formed teratomas containing tissues from all three germ layers, including bone and cartilage (mesoderm), neural rosettes (ectoderm), and ciliated respiratory epithelia (endoderm), among others (Fig. 3b).

Although stable putative ES stem lines were generated in only four of the six experiments, numerous other blastomere-derived outgrowths contained cells with both embryonic and extraembryonic stem-cell-like morphology. When FGF-4 was added to the media, seven putative TS lines were established, which maintained normal karyotype and expressed markers of TS cells (Fig. 2b, d, f, h, j). These cells were negative for Oct-4 (Fig. 2h) and for $\alpha$-feto protein. RT-PCR analysis confirmed that these cells expressed Cdx2, but not Oct-4; nanog and Rex-1 were expressed in both the putative TS and ES cell lines (data not shown). Putative TS cells contributed to the extraembryonic lineage in chimaeric fetuses generated by aggregation with the LacZ ${ }^{+}$TS cells (Supplementary Fig. 2).

In two control experiments, individual blastomeres $(n=44)$ isolated from 8-cell embryos were plated into 20-100- $\mu$ l drops

${ }^{1}$ Advanced Cell Technology, Worcester, Massachusetts 01605, USA. ${ }^{2}$ University of Wisconsin, State Laboratory of Hygiene, Molecular Cytogenetics, Madison, Wisconsin 53706, USA. ${ }^{3}$ Department of Pathology and Laboratory Medicine, University of Wisconsin Medical School, Madison, Wisconsin 53706, USA. ${ }^{4}$ Institute for Regenerative Medicine, Wake Forest University School of Medicine, Winston-Salem, North Carolina 27157, USA.

*These authors contributed equally to this work. 
containing mES cell culture medium. Most of the blastomeres failed to divide over the 10-day period of culture, whereas $9(20 \%)$ generated small clusters of differentiated trophoblast-like giant cells (Fig. 1g, h) before arresting at the 2-6-cell stage, thus suggesting that cell co-culture was critical to the success of this system.

The blastomere-biopsied embryos in the present study developed to term without a reduction in their developmental capacity $(49 \%$ (23 out of 47) live young versus $51 \%$ (38 out of 75) for control nonbiopsied embryos $\left(\chi^{2}\right.$ test, $\left.\left.P=0.85\right)\right)$. These results are consistent with human data, which indicate that normal and pre-implantation genetic diagnosis (PGD)-biopsied embryos develop into blastocysts with comparable efficiency ${ }^{12}$.

Although only 36 out of 125 blastomeres (29\%) generated inner cell mass-like outgrowths, and only 12 stable putative ES and TS cell
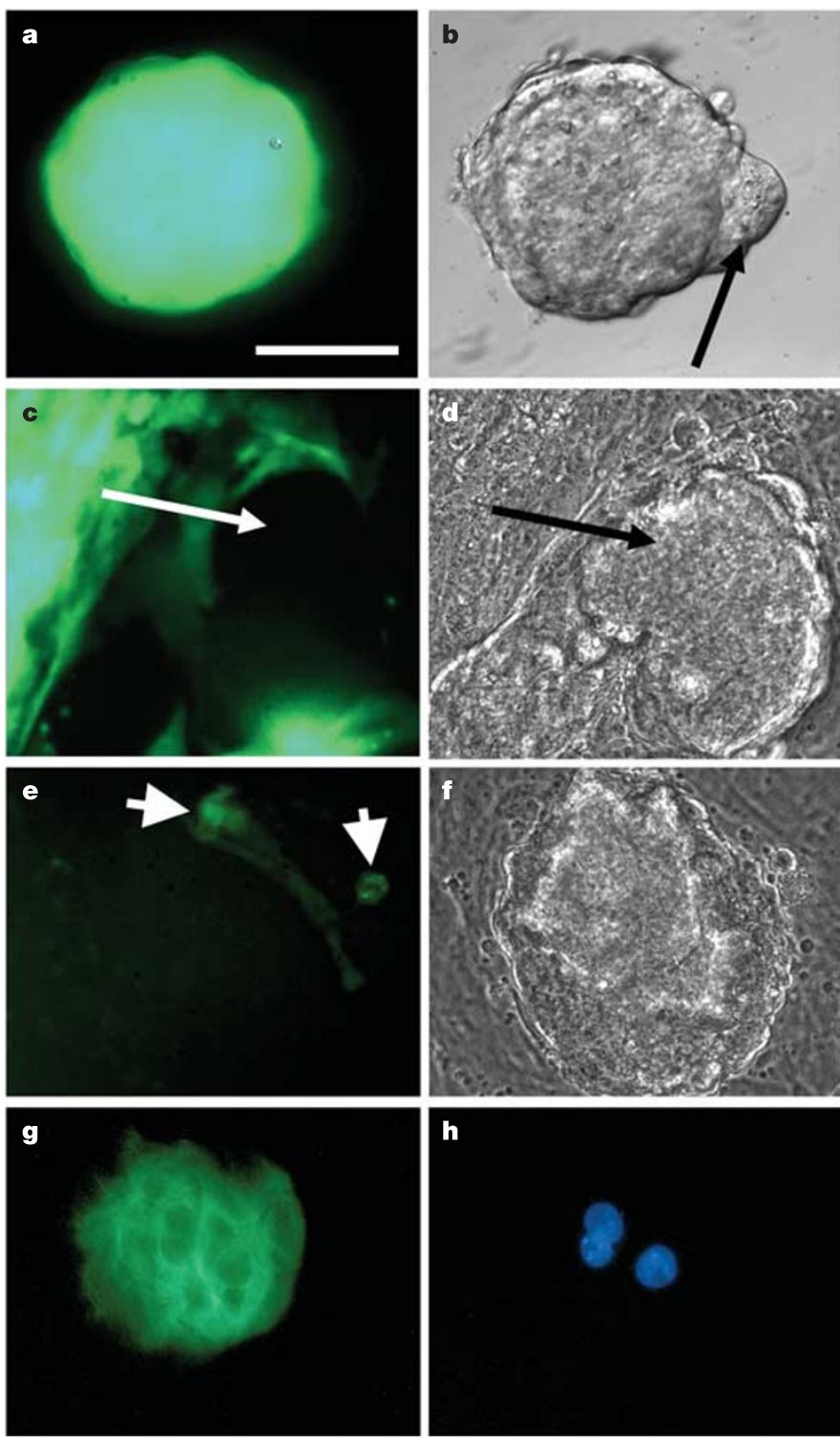

Figure 1 Stages of single blastomere growth in the presence or absence of mES cells. a, b, Clump of GFP-positive mES cells $48 \mathrm{~h}$ after aggregation with single blastomeres; arrow shows a protruding cluster of GFP-negative cells. c, d, Outgrowth of GFP-negative cells aggregated with GFP-positive mES cells, after being plated on MEFs; arrows point to GFP-negative cells.

e, $\mathbf{f}$, Passage 1 of the outgrowth; arrows show remaining GFP-positive mES cells. $\mathbf{g}, \mathbf{h}$, Single blastomere outgrowth on MEFs for 4 days without ES cells, stained with Troma-1 and DAPI. a, c, e, Green fluorescence; b, Hoffman modulation optics; $\mathbf{d}, \mathbf{f}$, phase contrast. Scale bar, $100 \mu \mathrm{m}$. lines were generated in this study (compared to approximately $25-35 \%$ for normal mouse blastocysts), we believe that this success rate can be considerably increased by greater attention to the earliest stages of blastomere outgrowth, as well as the use of various measures that influence the spontaneous differentiation of pluripotent ES cells into trophectoderm and other cell types. Owing to the labour intensiveness of the various steps, only conspicuous outgrowths were selected for further passaging, although many of the GFPpositive ES clusters were observed to contain other blastomerederived stem cells. Preliminary experiments using all blastomeres at the 8-cell stage indicate that most of the blastomeres give outgrowths
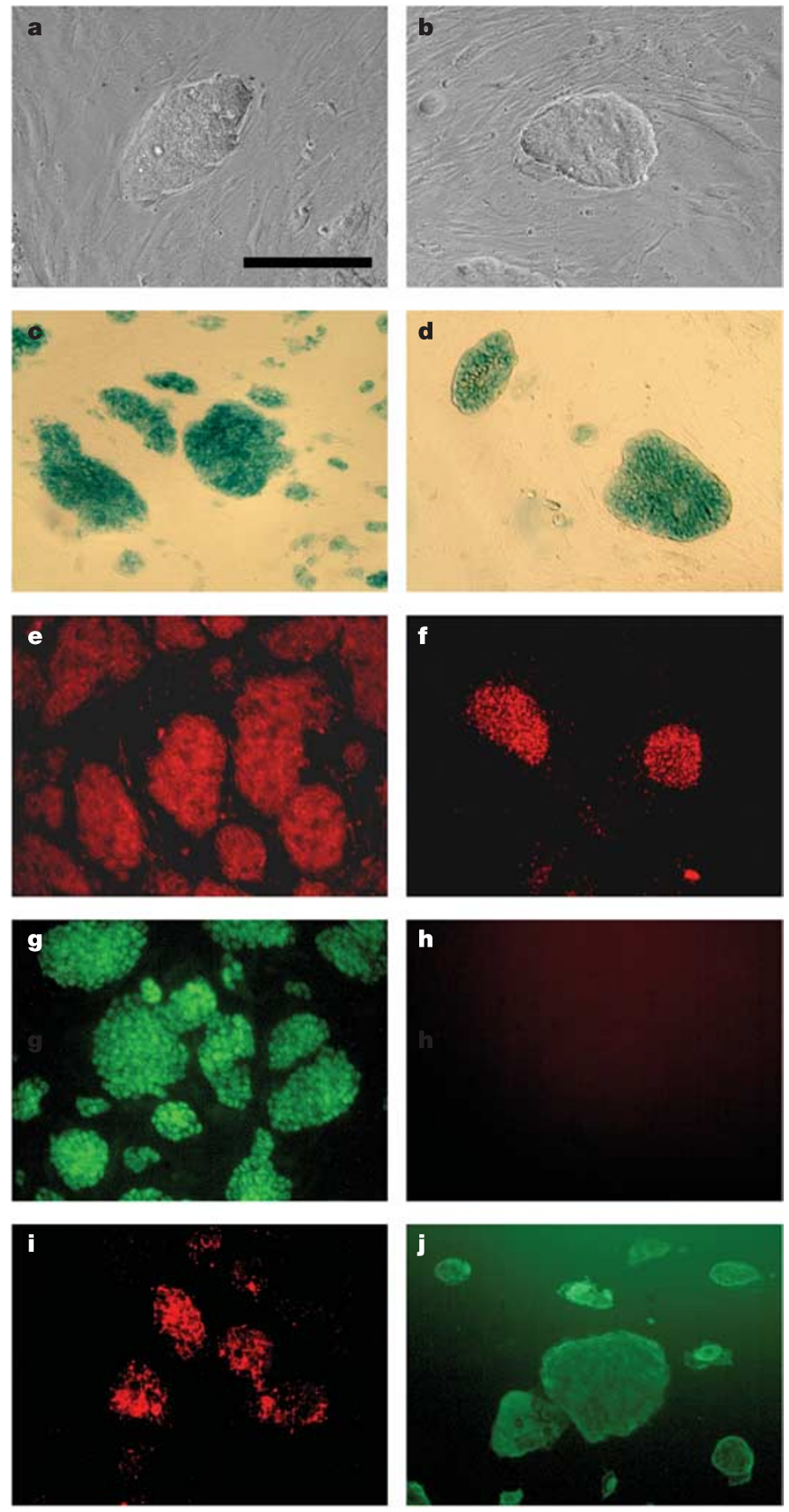

Figure 2 Comparison of putative ES (left column) and TS (right column) cell lines derived from single blastomeres. a, b, Phase contrast photograph of typical colonies. c, d, LacZ-stained colonies, showing their single blastomere origin. e, f, Alkaline phosphatase staining. $\mathbf{g}$, h, Indirect immunofluorescence with antibodies to Oct-4. i, SSEA-1 staining of putative ES cells; j, Troma-1 staining of putative TS cells (same field as $\mathbf{h}$ ). Scale bar, $200 \mu \mathrm{m}$. 
that appear to be morphologically identical. However, it is unclear whether the individual blastomeres have different developmental fates based on these observations. The microenvironment is clearly critical, and variables such as ES cell number, the spatial orientation of blastomeres, ES cells and MEFs, as well as media composition, seem to be important.

Previous studies have described different approaches to establish pluripotent stem cell lines from blastocysts ${ }^{3-5}$ and 8-20-cell stage morula $e^{6-9,13,14}$. In two studies, stem cell lines were established from disaggregated morulae, although the cells from the embryos were cultured together and free to reaggregate ${ }^{9,13}$. However, none of these strategies uses single isolated cells that could be used in conjunction with assisted reproduction technologies such as PGD, and, importantly, that would not interfere with the embryo's normal development to birth. There have been previous attempts in both humans and animals to induce single isolated blastomeres to proliferate in vitro, although none of these investigators derived pluripotent stem cell lines. One study ${ }^{15}$ cultured individual biopsied
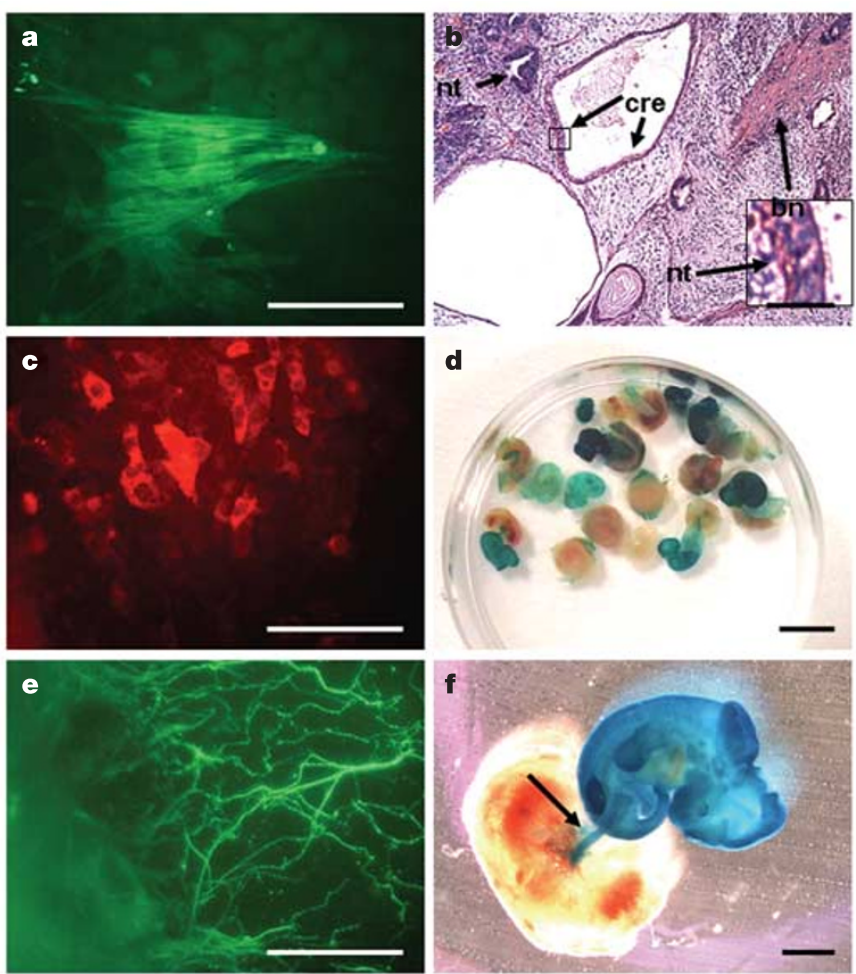

$\mathbf{g}$

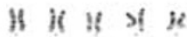
it it it it is
Y1 4 it 4 is
u $x$ \& $y$ f.
It it it by
if it if 0
of 4 a 4 औ
4 \& 4 a 11

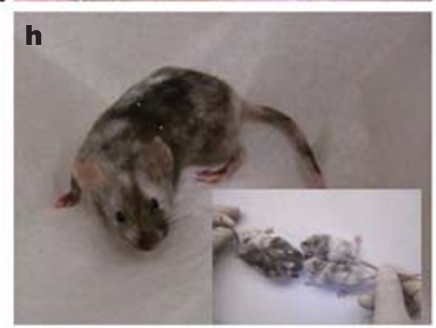

Figure 3 | Differentiation of blastomere-derived $\mathrm{mES}$ cells in vitro and in vivo. a, c, e, Immunofluorescence analysis of molecular markers of mesoderm (muscle actin, a), primitive endoderm ( $\alpha$-feto protein, $\mathbf{c}$ ) and ectoderm ( $\beta$ III tubulin, e). $\mathbf{g}$, Representative chromosome spreads of two single-blastomere-derived mES cell lines. $\mathbf{b}$, Teratomas section, stained with haematoxylin and eosin. bn, bone (mesoderm); nt, neural tissue (ectoderm); cre and insert, ciliated respiratory epithelium (endoderm). d, f, h, 11.5 d.p.c. chimaeric embryos $(\mathbf{d}, \mathbf{f})$ and chimaeric pups $(\mathbf{h})$ produced from three $\mathrm{mES}$ cell lines; arrow in $\mathbf{f}$ shows the extraembryonic mesoderm-derived placental labyrinth, also chimaeric. Scale bars: a, c, $100 \mu \mathrm{m}$; e, $200 \mu \mathrm{m}$; d, $10 \mathrm{~mm}$; f, $2 \mathrm{~mm}$. mouse blastomeres in vitro on different extracellular matrix components, including fibronectin and laminin, which have been shown to promote attachment and proliferation of blastomeres in vitro and to enhance blastocyst development in various animal species $^{16-18}$. Most of the isolated blastomeres divided to form small sheets of 6-8 cells with a trophoblastic morphology similar to that described in ref. 19. In the human, another study ${ }^{20}$ observed proliferation of blastomeres that had been removed and co-cultured with the biopsied embryos (range 1-8 cells per blastomere), although in all cases differential labelling indicated that they had generated trophectodermal cells. Unfortunately, the molecular mechanisms regulating these events are poorly understood, and it is unclear whether the success of the ES co-culture system in the present study is attributable to substances secreted by the ES cells or if cell-cell contact is required.

The developmental capacity of blastomeres isolated from mammalian embryos has been studied extensively, and it is clear that they retain their pluripotency, and, indeed, are capable of regular in vivo development upon transfer into mice ${ }^{21}$, rabbits ${ }^{22}$, sheep ${ }^{23}$, swine $e^{18,24,25}$ and primates ${ }^{26}$. We have demonstrated that single pre-implantation blastomeres can also be used to establish embryonic and extraembryonic stem cell lines using an approach that does not interfere with the developmental potential of the parent embryo. The biopsy procedure described here is carried out in IVF clinics worldwide without a reduction in pregnancy rate. However, further investigations are required to determine whether stem cell lines can be derived from other mammalian species, including humans, using this micromanipulation technique. The ability to generate human ES cells from PGD blastomeres could circumvent the ethical concerns voiced by many, and allow the banking of autologous ES cell lines for children born from transferred embryos.

\section{METHODS}

Generation of ES and TS cell lines. Eight-cell stage 129/Sv-ROSA26:lacZ mouse embryos were biopsied through a hole in the zona pellucida using piezo-pulse drilling. The biopsied embryos were transferred to the oviducts of 1.5 d.p.c. synchronized surrogates, and each separated blastomere was aggregated with a small clump ( $\leq 100$ cells) of GFP-positive 129 Sv/CD- $1 \mathrm{mES}$ cells in a $300-\mu \mathrm{m}$ depression created by pressing a needle into the bottom of a plastic tissue culture plate, as described ${ }^{11}$. After incubation for $24-48 \mathrm{~h}$ in mES cell growth medium ${ }^{11}$ supplemented with $2,000 \mathrm{U} \mathrm{ml}^{-1}$ mouse leukaemia inhibitory factor (LIF; Chemicon) and $50 \mu \mathrm{M}$ MEK1 inhibitor (Cell Signaling Technology), a growing bud of GFP-negative cells was observed on the sides of the majority of GFP-mES clusters. The aggregates were plated onto mitomycin C-treated MEFs and cultured in mES cell growth medium until GFP-negative clumps became large enough for dispersion ( $>20$ cells), which then were separated from GFP-positive $\mathrm{mES}$ cells by hand with a microcapillary under a fluorescence microscope. The cells were dissociated and expanded by alternating mechanical dissociation and digestion with $0.05 \%$ trypsin (Invitrogen). Blastomere outgrowths that morphologically resembled trophoblast and extraembryonic endoderm but not ES cells were further cultured in the mES cell medium with $50 \mathrm{ng} \mathrm{ml}^{-1} \mathrm{FGF}-4$, and produced TS-like cells that were maintained under these conditions and passaged with trypsin.

Immunofluorescence and alkaline phosphatase staining. Indirect immunofluorescence staining was performed on cells growing on 4-well tissue culture plates as previously described ${ }^{2,5}$. The following primary antibodies were used: Oct-4 (Santa Cruz Biotechnology), SSEA-1 (developed by D. Solter and B. Knowles and obtained through the DSHB (Developmental Studies Hybridoma Bank) of the University of Iowa), Troma-1 (raised by P. Brulet and R. Kemler and obtained through DSHB), $\alpha$-feto protein (DACO), $\beta$ III tubulin (Covance) and muscle actin (Abcam). Alkaline phosphatase staining was performed using the Vector red kit from Vector Laboratories.

Chimaeras and X-gal staining. Approximately 15 ES cells or TS cells were injected either into the blastocoels of expanded blastocysts or into the perivitelline space of pre-compacting 8-cell stage embryos obtained from CD-1 mice. Blastocyst chimaeras were transferred into the uteri of 2.5 d.p.c. surrogate mice $4 \mathrm{~h}$ after the ES cell injections. Most of the 8-cell stage chimaeric embryos developed into expanded blastocysts after overnight culture in mES medium, and were then transferred to surrogate mice. The pregnancies were terminated at days $11.5,12.5$ or 19.5 , and the embryos and placentas were fixed overnight in $4 \%$ 
paraformaldehyde and washed with PBS overnight; LacZ staining using a X-gal staining kit (GAL-S, Sigma) was done to test for chimaerism in the embryos. Teratomas. Approximately 1 million ES cells were injected into the rear thigh of a NOD-SCID mouse. After approximately 2 months the mice were killed and the teratomas excised, fixed in $4 \%$ paraformaldehyde, embedded in paraffin and sectioned.

Received 18 August; accepted 4 October 2005.

Published online 16 October 2005.

1. Committee on the Biological and Biomedical Applications of Stem Cell Research, Board on Life Sciences, National Research Council, Board on Neuroscience and Behavioral Health, Institute of Medicine (ed.) Stem Cells and the Future of Regenerative Medicine (National Academies Press, Washington DC, 2002).

2. Lanza, R. et al. (eds) Handbook of Stem Cells. Vol 1: Embryonic Stem Cells (Elsevier/Academic Press, San Diego, California, 2004).

3. Evans, M. J. \& Kaufman, M. H. Establishment in culture of pluripotential cells from mouse embryos. Nature 292, 154-156 (1981).

4. Thomson, J. A. et al. Embryonic stem cell lines derived from human blastocysts. Science 282, 1145-1147 (1998).

5. Cowan, C. A. et al. Derivation of embryonic stem-cell lines from human blastocysts. N. Engl. J. Med. 350, 1353-1356 (2004)

6. Strelchenko, N. et al. Morula-derived human embryonic stem cells. Reprod. BioMed. 9, 623-629 (2004).

7. Sukoyan, M. A. et al. Embryonic stem cells derived from morulae, inner cell mass, and blastocysts of mink: comparisons of their pluripotencies. Mol. Reprod. Dev. 36, 148-158 (1993).

8. Mitalipova, M., Beyhan, Z. \& First, N. L. Pluripotency of bovine embryonic cell line derived from precompacting embryos. Cloning 3, 59-67 (2001).

9. Delhaise, F. et al. Establishment of an embryonic stem cell line from 8-cell stage mouse embryos. Eur. J. Morphol. 34, 237-243 (1996).

10. Handyside, A. H. et al. Pregnancies from biopsied human preimplantation embryos sexed by Y-specific DNA amplification. Nature 344, 768-770 (1990).

11. Nagy, A., Gertsenstein, M., Vintersten, K. \& Behringer, R. (eds) Manipulating the Mouse Embryo: A Laboratory Manual (Cold Spring Harbor Laboratory Press, Cold Spring Harbor, New York, 2002).

12. Staessen, C. et al. Comparison of blastocyst transfer with or without preimplantation genetic diagnosis for aneuploidy screening in couples with advanced maternal age: a prospective randomized controlled trial. Hum. Reprod. 19, 2849-2858 (2004)

13. Eistetter, H. R. Pluripotent embryonal stem cell lines can be established from disaggregated mouse morulae. Dev. Growth Differ. 31, 275-282 (1989).

14. Tesar, P. J. Derivation of germ-line-competent embryonic stem cell lines from preblastocyst mouse embryos. Proc. Natl Acad. Sci. USA 102, 8239-8244 (2005).

15. Wilton, L. \& Trounson, A. Biopsy of preimplantation mouse embryos: development of micromanipulated embryos and proliferation of single blastomeres in vitro. Biol. Reprod. 40, 145-152 (1989).

16. Tao, T. \& Niemann, H. Cellular characterization of blastocysts derived from rabbit 4-, 8- and 16-cell embryos and isolated blastomeres cultured in vitro. Hum. Reprod. 15, 881-889 (2000).

17. Armant, D. R., Kaplan, H. A. \& Lernarz, W. J. Fibronectin and laminin promote in vitro attachment and outgrowth of mouse blastocysts. Dev. Biol. 116, 519-523 (1986).

18. Saito, S. \& Niemann, H. Effects of extracellular matrices and growth factors on the development of isolated porcine blastomeres. Biol. Reprod. 44, 927-936 (1991).

19. Tarkowski, A. K. \& Wroblenska, J. Development of blastomeres of mouse eggs isolated at the 4- and 8-cell stage. J. Embryol. Exp. Morphol. 18, 155-180 (1967).

20. Geber, S., Winston, R. M. L. \& Handyside, A. H. Proliferation of blastomeres from cleavage stage human embryos in vitro: an alternative to blastocyst biopsy for preimplantation diagnosis. Hum. Reprod. 10, 1492-1496 (1995).

21. Rossant, J. Postimplantation development of blastomeres isolated from 4- and 8-cell mouse eggs. J. Embryol. Exp. Morphol. 36, 283-290 (1976).

22. Moore, N. W., Adams, C. E. \& Rowson, L. E. Developmental potential of single blastomeres of the rabbit egg. J. Reprod. Fertil. 17, 527-531 (1968).

23. Willadsen, S. M. The development capacity of blastomeres from 4- and 8-cell sheep embryos. J. Embryol. Exp. Morphol. 15, 165-172 (1981).

24. Menino, A. R. \& Wright, R. W. Effect of pronase treatment, microdissection, and zona pellucida removal on the development of porcine embryos and blastomeres in vitro. Biol. Reprod. 28, 433-446 (1983).

25. Niemann, H. \& Reichelt, B. Manipulating early pig embryos. J. Reprod. Fertil. Suppl. 48, 75-94 (1993).

26. Chan, A. W. S. et al. Clonal propagation of primate offspring by embryo splitting. Science 287, 317-319 (2000).

Supplementary Information is linked to the online version of the paper at www.nature.com/nature.

Acknowledgements The monoclonal antibodies to SSEA-1 (developed by D. Solter and B. Knowles) and to Troma1 (developed by P. Brulet and R. Kemler) were obtained from the Developmental Studies Hybridoma Bank developed under the auspices of the NICHD and maintained by The University of lowa.

Author Information Reprints and permissions information is available at npg.nature.com/reprintsandpermissions. The authors declare competing financial interests: details accompany the paper at www.nature.com. Correspondence and requests for materials should be addressed to R.L. (rlanza@advancedcell.com). 